\title{
Substituição de Amianto por Silicato de Alumínio e Grafite Expansível em Compósito de Poliuretano utilizado em Motor-foguete
}

\author{
Henrique Crespim, Margarete F. P. Azevedo, Luis H. David, Silvana N. Cassu, Vera L. Lourenço \\ Divisão de Química, IAE, Comando-Geral de Tecnologia Aeroespacial (CTA)
}

Resumo: Compósitos de poliuretano e amianto (liner) são utilizados como revestimento interno em paredes de motor-foguete, conferindo proteção térmica e garantindo a adesão entre o propelente e as paredes do motor. No entanto, o uso do amianto tem sido restringido devido à sua toxidade. No presente trabalho, o amianto foi substituído por um silicato de alumínio hidratado (SA) e pelo grafite expansível (GE) em diferentes teores no liner. Resultados de análise termogravimétrica (TG) mostraram que a estabilidade térmica do liner praticamente não é afetada pela substituição das cargas, embora a energia de ativação (Ea) obtida para a decomposição tenha mudado, mostrando maiores valores para as amostras contendo as cargas SA e GE. A análise termomecânica (TMA) mostrou que o coeficiente de expansão térmica linear do liner contendo SA foi menor que aquele encontrado para o liner contendo amianto. O liner contendo a carga SA também apresentou os maiores valores de tensão nos testes mecânicos de tração.

Palavras-chave: Compósito de poliuretano, silicato de alumínio, grafite expansível, estabilidade térmica.

\section{Substitution of Asbestos for Aluminosiliacate and Expandable Graphite in Polyurethane Composites used in Rocket Motors}

Abstract: Composites of polyurethane (PU) and asbestos (liner) are used as internal coating of rocket motors, providing thermal protection and assuring the adhesion between propellant and the motor walls. However, the use of asbestos has been restricted due to its hazardous nature. In the present work, asbestos was replaced by hydrated alumina silicate (SA) and expandable graphite (GE) in different contents. Thermogravimetric analysis (TG) showed that the thermal stability of liners was practically unaffected by the filler replacement although the activation energy obtained for the decomposition has changed. Thermomechanical analysis (TMA) showed that coefficients of thermal expansion of SA/liners were lower than asbestos/liner. SA/liners also presented the highest tension values in mechanical tests.

Keywords: Polyurethane composite, aluminosilicate, expandable graphite, thermal stability.

\section{Introdução}

Poliuretanos elastoméricos apresentam como características intrínsecas a alta resistência ao impacto e a boa adesão a superfícies compatíveis com a natureza química da cadeia de poliuretana utilizada ${ }^{[1]}$. Este material tem sido usado, entre outras aplicações, como revestimento interno em paredes de motor-foguete. Neste caso, é utilizado um compósito de poliuretano contendo cargas como, negro de fumo e amianto, que recebe o nome de "liner". O liner utilizado em motor-foguete tem como principais funções garantir a adesão entre o propelente sólido e a estrutura metálica do motor e atuar como camada ablativa durante a queima do propelente, quando a temperatura varia entre 2000 e $4000{ }^{\circ} \mathrm{C}^{[2]}$. Apesar da exposição a altas temperaturas, o tempo de exposição da camada de liner é da ordem de segundos. O processo de ablação é alcançado por meio da transferência auto-regulada de massa e de calor envolvendo um isolante com baixa condutividade térmica, pirólise e concomitante formação de uma camada refratária com alto teor de carbono na superfície do isolante (Figura 1) ${ }^{[2-4]}$. A baixa condutividade térmica combinada aos curtos tempos de exposição à chama, reduz o aquecimento por condução de calor das partes protegidas ${ }^{[5]}$. Acima da temperatura de decomposição, o isolante produz gases a partir da pirólise na zona de pirólise e essa camada se degrada, resultando em um material rico em carbono. A presença da camada rica em carbono regula a penetração do fluxo de calor a partir da superfície e produz um gradiente decrescente de temperatura. Os gases da pirólise e a expansão térmica geram tensão na camada rica em carbono e se não reforçado, o material degrada facilmente aumentando a taxa de erosão da superfície ${ }^{[4]}$. Por esta razão é necessário que sejam adicionados reforços à matriz polimérica, e neste caso são utilizadas cargas inorgânicas visando aumentar a resistência mecânica e térmica do liner. A aparência de uma amostra de liner após teste em banco de provas é exemplificada na Figura 2, onde é mostrado parte da superfície do liner padrão preparado com amianto tipo crisotila. Nesse

Autor para correspondência: Vera L. Lourenço, Instituto de Aeronáutica e Espaço, Divisão de Química, Comando-Geral de Tecnologia Aeroespacial, Praça Mal. Eduardo Gomes 50, CEP: 12228-904, São José dos Campos, São Paulo, SP, Brasil. E-mail: vlucia@iae.cta.br 

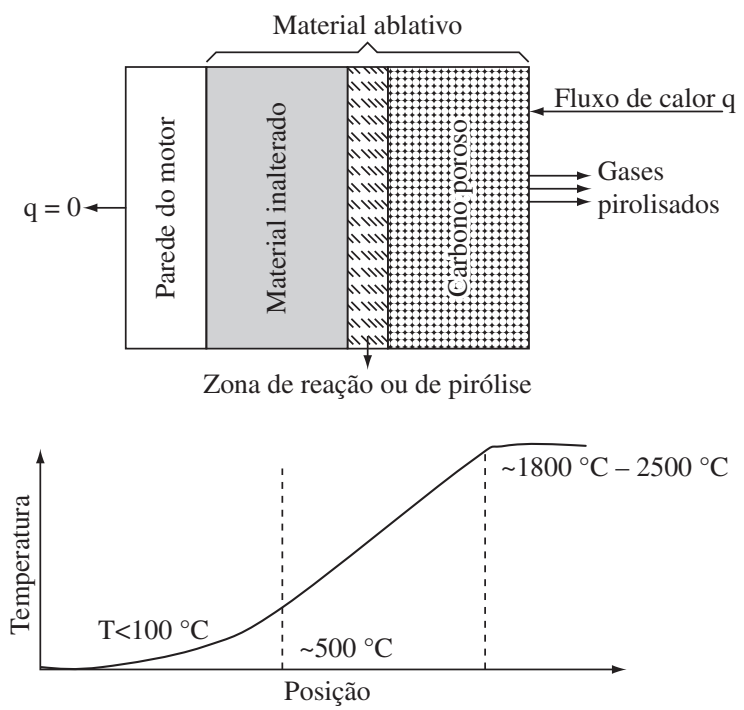

Figura 1. Evolução do processo ablativo.

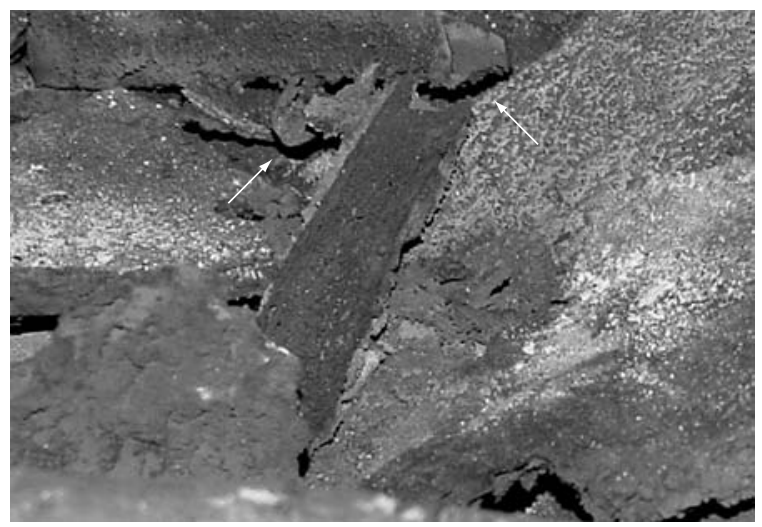

Figura 2. Fotografia da superfície da camada de liner após a queima do motor-foguete. As setas indicam as delaminações superficiais geradas em decorrência do processo ablativo (ampliado cinco vezes).

tipo de teste um motor-foguete de cerca de $700 \mathrm{~kg}$ é queimado e alguns parâmetros podem ser analisados, entre eles, o desempenho da camada de liner. A Figura 2 mostra uma superfície que sofreu ablação, observando-se algumas delaminações decorrentes desse processo.

As cargas convencionalmente utilizadas no liner aplicado em motores-foguete produzidos no Brasil são o negro de fumo e o amianto tipo crisotila $\left(\mathrm{Mg}_{3}\left(\mathrm{Si}_{2} \mathrm{O}_{5}\right)(\mathrm{OH})_{4}\right)$. A adição do negro de fumo visa aumentar o teor de carbono no material, e consequentemente, a espessura da camada ablativa. O amianto é utilizado para melhorar a resistência mecânica e térmica. No entanto, a utilização do amianto foi banida nos últimos anos em vários países devido à sua toxicidade, e temse atribuído doenças pulmonares como asbestose, câncer de pulmão e mesotelioma à exposição aos diferentes tipos de amianto, inclusive o tipo crisotila ${ }^{[6-9]}$. No Brasil, desde 2001, o Instituto Brasileiro de Defesa do Consumidor (IDEC) juntamente aos Ministérios do Trabalho e da Saúde vem trabalhando para banir o uso do amianto ${ }^{[10]}$.

Neste trabalho, o amianto foi substituído pelo algamatolito, um silicato de alumínio hidratado $\left(\mathrm{Al}_{2} \mathrm{O}_{3} \cdot 4 \mathrm{SiO}_{2} \cdot \mathrm{H}_{2} \mathrm{O}\right)$ encontrado em minas brasileiras, contendo como principais componontes a pirofilita $\left.\left(\mathrm{Si}_{4} \mathrm{O}_{10}\right) \mathrm{Al}_{2}(\mathrm{OH})_{2}\right)$ e a muscovita $\left(\mathrm{KAl}_{2}\left[(\mathrm{OH})_{2} / \mathrm{AlSi}_{3} \mathrm{O}_{10}\right)^{[11]}\right.$. O grafite expansível também foi utilizado como carga, tendo como finalidade aumentar o teor de carbono presente no liner e causar uma redução na transferência de calor durante o processo de expansão desse material causado pelo aquecimento. Os compósitos preparados com estas cargas foram testados e comparados ao liner contendo amianto, que é o utilizado atualmente em motores-foguete, em relação às propriedades térmicas e mecânicas.

\section{Experimental}

Os compósitos de poliuretano (liner) foram preparados utilizando-se o polibutadieno líquido hidroxilado (PBLH-Petroflex-H) e as cargas negro de fumo (NF), amianto (Am, OB 80303, Minérios Ouro Branco), silicato de alumínio hidratado (SA, Fleximil Plus da Lamil) e grafite expansível (GE, da Nacional de Grafite Ltda.) nos teores apresentados na Tabela 1. As misturas foram mantidas sob vácuo para remoção dos gases antes da reação de cura, após o que foi adicionado o diisocianato de tolueno (TDI, Isociantos do Brasil). As misturas ainda líquidas foram colocadas em moldes de aço inox e levadas à estufa a $50{ }^{\circ} \mathrm{C}$ por sete dias para completar a reação de cura.

O analisador simultâneo SDT-Q600 da TA Instruments foi utilizado para a obtenção de análises termogravimétricas e calorimétricas, o que permitiu o acompanhamento em tempo real da decomposição térmica e da variação de fluxo de calor dos materiais puros (amianto, GE e SA) e das amostras de liner. As análises foram conduzidas na faixa de temperatura entre 25 e $1000{ }^{\circ} \mathrm{C}$ nas razões de aquecimento de 5, 10, 20 e $50{ }^{\circ} \mathrm{C} / \mathrm{min}$, sob atmosfera de ar sintético, ou a $10{ }^{\circ} \mathrm{C} / \mathrm{min}$ na mesma faixa de temperatura, sob atmosfera inerte de nitrogênio até $530{ }^{\circ} \mathrm{C}$, temperatura na qual a atmosfera de nitrogênio foi substituída por ar sintético.

Para a determinação do coeficiente de expansão térmica linear foi utilizado o TMA-943 Du Pont. Amostras de dimensões de cerca de $5 \times 5 \times 2 \mathrm{~mm}$ foram resfriadas até $-130{ }^{\circ} \mathrm{C}$ com nitrogênio líquido e aquecidas a $5{ }^{\circ} \mathrm{C} / \mathrm{min}$ até $60^{\circ} \mathrm{C}$ em atmosfera de nitrogênio sob carga de $1 \mathrm{~g}$.

O ensaio de tração foi conduzido a temperatura ambiente em uma máquina de ensaios Zwick 1474, segundo a ASTM D $412^{[13]}$, sendo ensaiados pelo menos 6 corpos de prova de cada compósito a uma velocidade de $500 \mathrm{~mm} / \mathrm{min}$.

As análises granulométricas das amostras de amianto e de grafite foram realizadas em uma máquina vibratória da

Tabela 1. Compósitos de poliuretanos (liner) contendo diferentes cargas.

\begin{tabular}{lcccc}
\hline \multicolumn{1}{c}{ Amostra } & NF (\%) & Am (\%) & SA (\%) & GE (\%) \\
\hline Liner/Am & 2,7 & 8,0 & - & - \\
Liner/SA 8 & 2,7 & - & 8,0 & - \\
Liner/SA 30 & 2,7 & - & 30,0 & - \\
Liner/SA 8/GE 8 & 2,7 & - & 8,0 & 8,0 \\
Liner/SA 30/GE 8 & 2,7 & - & 30,0 & 8,0 \\
\hline
\end{tabular}


Tabela 2. Valores de diâmetro médio (Dm) das cargas utilizadas nos compósitos de poliuretano.

\begin{tabular}{cc}
\hline Carga & Dm $(\mu \mathbf{m})$ \\
\hline Am & 1100 \\
SA & 11 \\
GE & 305 \\
\hline
\end{tabular}

marca Produtest, com tempo de vibração de 20 minutos e intensidade de vibração de 10 . Foi utilizado um conjunto de 11 peneiras entre 7 e 200 mesh, e as análises foram feitas em duplicatas. Como o silicato de alumínio apresentava uma granulometria muito fina, esta análise foi realizada no equipamento Mastersizer 2000 da Malvern, que utiliza espalhamento de laser para a determinação do tamanho das partículas. A análise foi realizada utilizando água como dispersante, sendo feita em triplicata. Os valores de diâmetro médio (Dm) das diferentes cargas é mostrado na Tabela 2.

\section{Resultados e Discussão}

O comportamento térmico das cargas utilizadas foi avaliado por análise termogravimétrica (TG) em atmosfera oxidante e os resultados obtidos são mostrados na Figura 3.

O grafite expansível tem sido utilizado como aditivo para aumentar a resistência a chama em materiais termopláti$\cos ^{[14-16]}$. Durante o aquecimento desse material, ocorre uma expansão de volume que pode chegar a 300 vezes o volume original das partículas. A rápida oxidação do carbono consome oxigênio reduzindo os teores disponíveis desse gás para a queima do material. Além disso, a expansão gera uma camada isolante ao calor, o que retarda a propagação da chama ${ }^{[14]}$. O

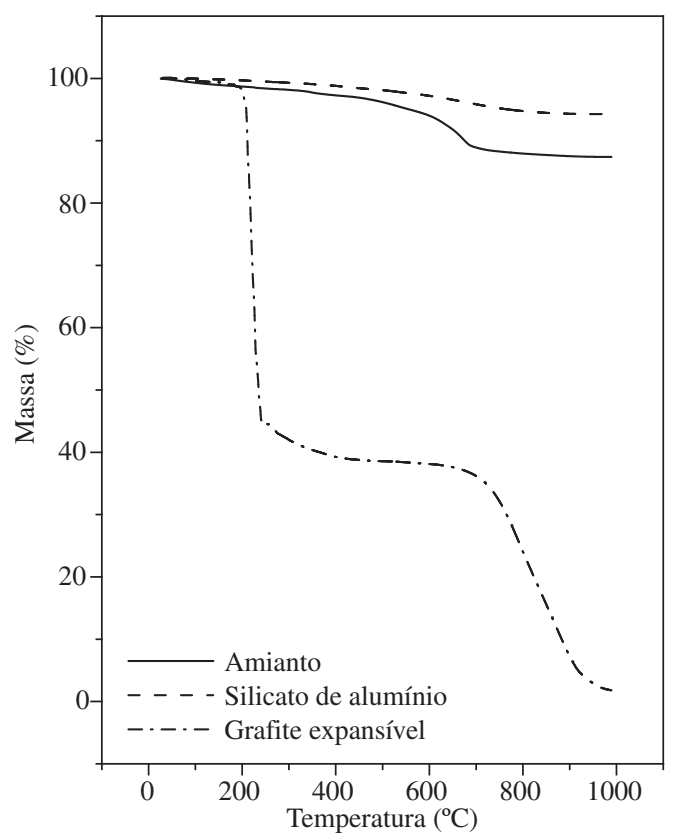

Figura 3. Curvas de massa a $10{ }^{\circ} \mathrm{C} / \mathrm{min}$ em ar sintético em função da temperatura para as diferentes cargas utilizadas no liner: (-) amianto, (---) silicato de alumínio, (----) grafite expansível. grafite expansível utilizado apresentou uma expansão de $21 \%$ em seu volume quando aquecido a $300{ }^{\circ} \mathrm{C}$ por 2 horas, de acordo com os resultados obtidos por análise granulométrica. A expansão ocorre em consequiência da liberação de voláteis acompanhada por uma perda de massa de cerca de $60 \%$ entre 200 e $700{ }^{\circ} \mathrm{C}$, como pode ser observado na Figura 3.

As curvas de perda de massa obtidas em ar sintético para o amianto e o silicato de alumínio também são mostradas na Figura 3. Tanto o amianto tipo crisotila como o silicato de alumínio mostram o início da perda de massa em cerca de $400{ }^{\circ} \mathrm{C}$, apresentando 12 e $6 \%$ em perda de massa, respectivamente, até $800{ }^{\circ} \mathrm{C}$. A perda de massa entre 600 e $800{ }^{\circ} \mathrm{C}$ é atribuída à reação de dehidroxilação em amianto ${ }^{[12]} \mathrm{e} e \mathrm{em}$ silicatos de alumínio ${ }^{[17]}$.

As análises termogravimétricas dos compósitos de PU foram realizadas em atmosfera de nitrogênio/ar sintético e em ar sintético (Figura 4). Nota-se que as amostras analisadas nas diferentes atmosferas aparecem em dois conjuntos deslocados em relação à temperatura, sendo que as amostras analisadas em ar sintético apresentam o início do principal estágio de perda de massa em temperatura ligeiramente superior $\left(\sim 410^{\circ} \mathrm{C}\right)$ à perda de massa observada em atmosfera de nitrogênio/ar sintético $\left(\sim 330^{\circ} \mathrm{C}\right)$ (Figura $\left.4 \mathrm{a}\right)$. As curvas de perda de massa obtidas para o PU sem carga, em atmosfera inerte e oxidante, são mostradas na Figura $4 b$, juntamente com as respectivas curvas de fluxo de calor. Por esta figura observa-se que o PU sem carga também apresenta o principal estágio de perda de massa em maior temperatura quando analisado em atmosfera oxidante. Os processos exotérmicos observados nas curvas de fluxo de calor são coincidentes com os processos de perda de massa e indicam que os mecanismos de degradação do PU nas diferentes atmosferas são distintos, como era esperado. Na degradação termo-oxidativa observa-se que há um ligeiro ganho de massa em $220^{\circ} \mathrm{C}$, que deve estar associado à oxidação do polímero, gerando uma fina camada ablativa em sua superfície. Essa camada rica em carbono deve ser a responsável pela maior temperatura na qual se observa o início da principal perda de massa do poliuretano puro. A temperatura observada para esse processo está de acordo com a faixa de temperatura $\left(250\right.$ a $\left.600{ }^{\circ} \mathrm{C}\right)$ descrita para pirólise de materiais gerando camada ablativa ${ }^{[18]}$.

Ainda em relação à Figura 4a, observa-se que a adição das diferentes cargas, SA, SA/GE, ou amianto, não altera o perfil de decomposição do compósito de PU no estágio inicial, sendo a decomposição nesta etapa governada, principalmente, pela degradação da matriz polimérica. A perda de massa que ocorre após a substituição da atmosfera de nitrogênio por ar sintético, é causada pela oxidação do negro de fumo presente em todas as formulações de liner.

A energia de ativação de decomposição (Ea) do liner contendo as diferentes cargas em ar sintético foi calculada a partir das curvas termogravimétricas, em diferentes razões de aquecimento de acordo com a ASTM E-1641-04 ${ }^{[19]}$. Para o cálculo dos valores de Ea foi utilizado um gráfico do logaritmo da razão de aquecimento em função do inverso da 


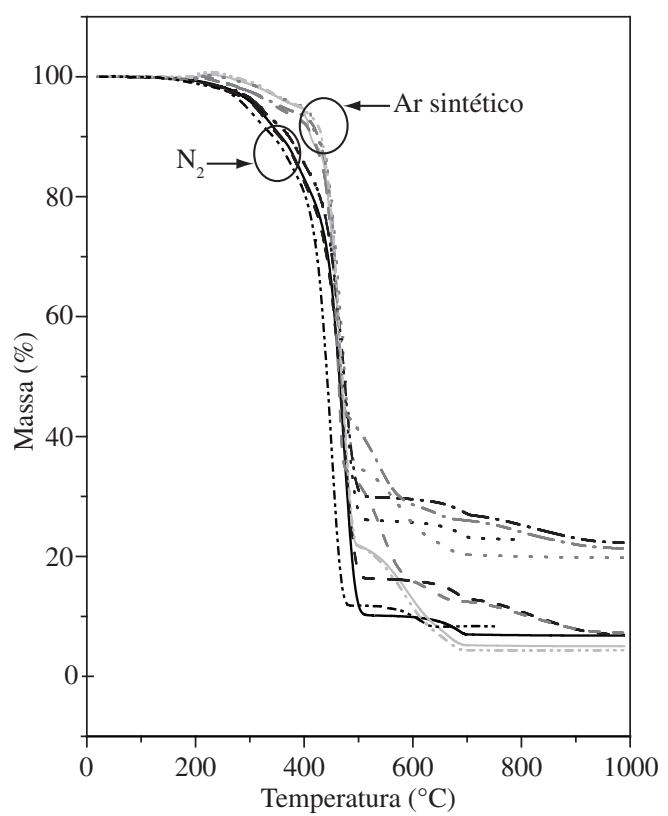

(a)

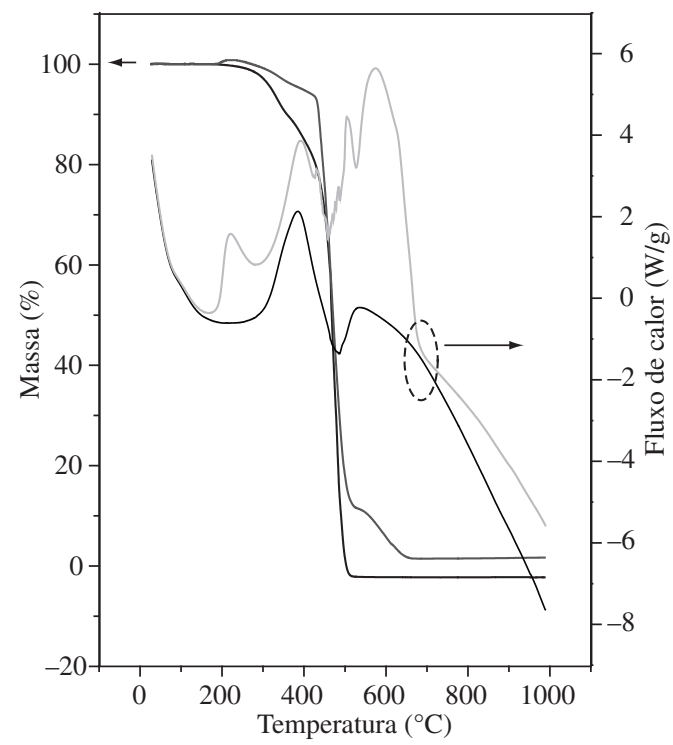

(b)

Figura 4. a) Curvas de massa em função da temperatura obtidas em atmosfera de $\mathrm{N}_{2}$ /ar sintético (curvas pretas) e ar sintético (curvas cinzas) para o liner contendo diferentes cargas: (-) SA8, (--) SA8/GE8, (...) SA30, (-•-.-) SA30/GE8, (-...-.) amianto; e b) Curvas de massa e fluxo de calor em função da temperatura para o PU em atmosfera $\mathrm{N}_{2}$ /ar sintético (curvas pretas) e ar sintético (curvas cinzas).

temperatura a $20 \%$ de conversão, como exemplificado na Figura 5a. A Ea foi obtida pelo coeficiente angular da reta (b), utilizando a expressão $b=-2,19 \mathrm{Ea} / \mathrm{R}$, onde $\mathrm{R}$ é a constante dos gases (Figura 5b). A Tabela 3 mostra os valores de Ea obtidos.

Observa-se que as amostras Liner/SA 8 e Liner/SA 8/GE 8 mostram os maiores valores de Ea para o início da decomposição térmica. A utilização do silicato de alumínio no lugar do amianto na mesma proporção (8\%) aumenta a energia de ativação para a decomposição de 74 para $81 \mathrm{~kJ} / \mathrm{mol}$, enquanto
Tabela 3. Valores de Ea, $\mathrm{Tg}$ e $\alpha$ para liner contendo diferentes cargas.

\begin{tabular}{lccc}
\hline \multicolumn{1}{c}{ Amostra } & $\mathbf{E a}(\mathbf{K J} / \mathbf{m o l})$ & $\mathbf{T g}\left({ }^{\circ} \mathbf{C}\right)$ & $\alpha\left(\mu \mathbf{m} / \mathbf{m}^{\circ} \mathbf{C}\right)$ \\
\hline Liner sem carga & 63 & $-57 \pm 1$ & $276 \pm 2$ \\
Liner/amianto & 74 & $-57 \pm 1$ & $391 \pm 8$ \\
Liner/SA 8 & 81 & $-61 \pm 4$ & $250 \pm 3$ \\
Liner/SA 30 & 65 & $-58 \pm 1$ & $222 \pm 4$ \\
Liner/SA 8/GE 8 & 98 & $-66 \pm 2$ & $313 \pm 12$ \\
Liner/SA 30/GE 8 & 62 & $-62 \pm 3$ & $256 \pm 15$ \\
\hline
\end{tabular}

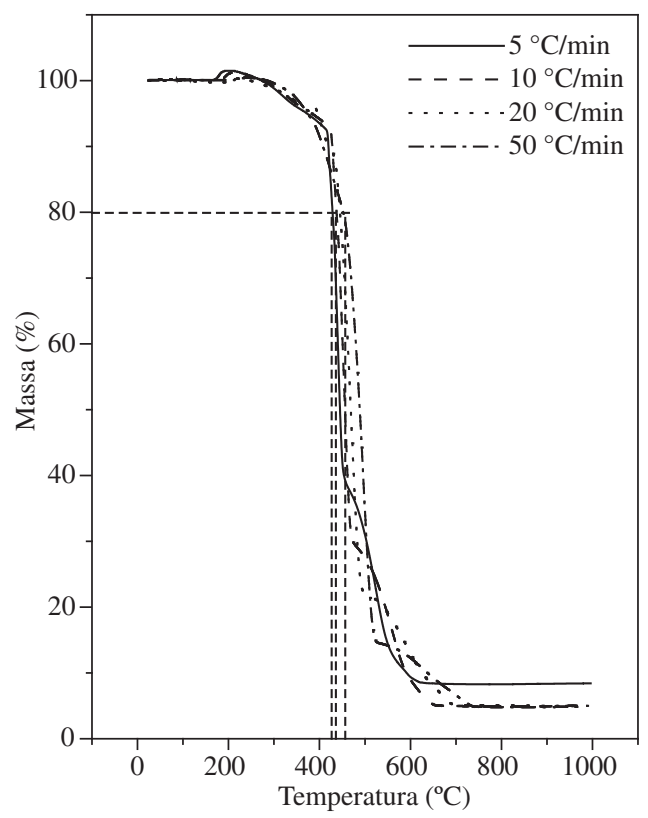

(a)

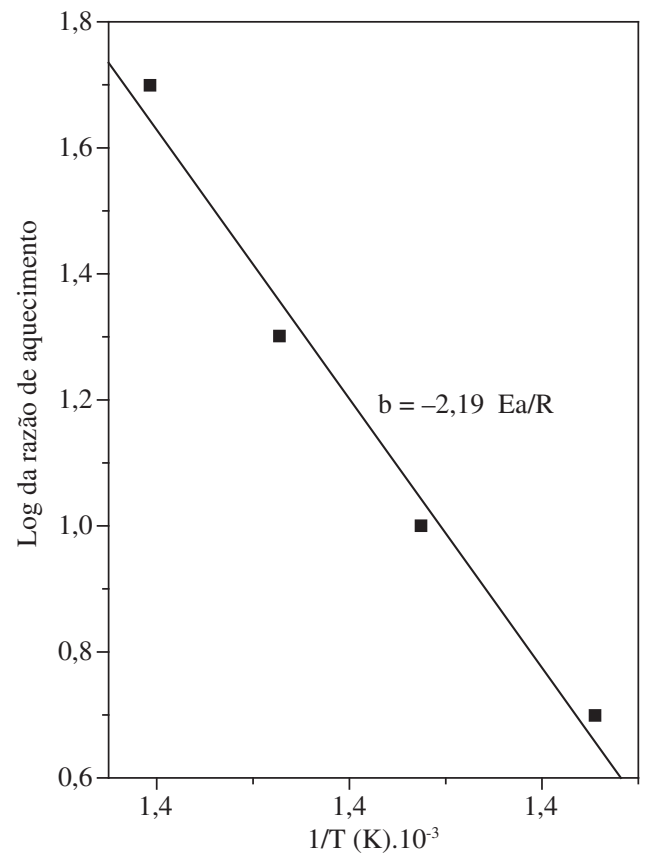

(b)

Figura 5. a) Exemplo da obtenção dos valores de temperatura a $20 \%$ de conversão para o cálculo de Ea; e b) gráfico utilizado para obtenção dos valores de Ea. 
que a adição de GE a essa formulação aumenta em $32 \%$ esse valor, que passa para $98 \mathrm{~kJ} / \mathrm{mol}$. A utilização de $30 \%$ de SA não altera o valor de Ea em relação ao PU sem carga, o que pode estar relacionado à melhor condução térmica em amostras contendo maiores teores de silicato de alumínio. Esses resultados mostram que tanto o SA como a mistura SA/GE podem ser utilizados em substituição ao amianto, desde que nas proporções adequadas, causando um aumento na energia de ativação para início da decomposição e, portanto, retardando o início da decomposição térmica.

Os valores de temperatura de transição vítrea $(\mathrm{Tg})$ e coeficiente de expansão térmica linear $(\alpha)$ obtidos para o liner contendo diferentes cargas também foram comparados. Esses valores foram obtidos por análise TMA e são mostrados na Tabela 2. Os valores de Tg apresentam uma pequena variação para menores temperaturas em amostras contendo as cargas SA e SA/GE em relação ao PU sem carga e ao Liner contendo amianto, o que não compromete o desempenho do liner contendo as cargas SA e SA/GE.

No caso do coeficiente de expansão térmica, é interessante que este seja o menor possível, pois as paredes metálicas do motor sofrem baixa expansão durante a variação de temperatura quando comparado aos polímeros contidos em seu interior (camada de liner e propelente sólido).

A utilização de $8 \%$ em massa da carga SA causou uma redução nos valores do coeficiente de expansão térmica de $36 \%$ em relação ao liner contendo amianto, sendo $9 \%$ menor também que o valor encontrado para o PU sem carga. O liner obtido com $30 \%$ de SA apresentou uma redução de $43 \%$ em relação ao liner contendo amianto. A adição do GE aos compósitos contendo 8 e $30 \%$ em massa de SA resultou em uma redução nos valores do coeficiente de expansão térmica de 20 e $34 \%$, respectivamente, em comparação ao liner contendo amianto. A redução no coeficiente de expansão térmica linear neste caso é menor que em relação às amostras contendo apenas o silicato de alumínio, já que a carga GE sofre expansão durante o aquecimento. Em relação a essa propriedade, todas as cargas testadas mostraram melhores valores quando comparados ao valor obtido para o liner padrão.

Os compósitos de PU foram avaliados por ensaios de tração (Figura 6). A utilização de $8 \%$ em massa de silicato de alumínio no compósito aumenta os valores de tensão na ruptura mantendo o mesmo alongamento conferido pelo amianto. A utilização de $30 \%$ em massa de silicato de alumínio aumenta ainda mais os valores de tensão na ruptura com pequena redução do alongamento em relação à amostra contendo $8 \%$ dessa carga. Por estes resultados observa-se que as partículas de silicato de alumínio conferem um maior reforço à matriz de PU em relação ao amianto. Este fato pode ser explicado pela diferença no tamanho das partículas de SA $(\sim 11 \mu \mathrm{m}) \mathrm{em}$ relação ao amianto, que apresenta forma fribilar e tamanho médio de $1100 \mu \mathrm{m}$ com larga distribuição. Além disso, as partículas de SA são distribuídas mais homogeneamente na matriz polimérica que as fibras de amianto, que em alguns

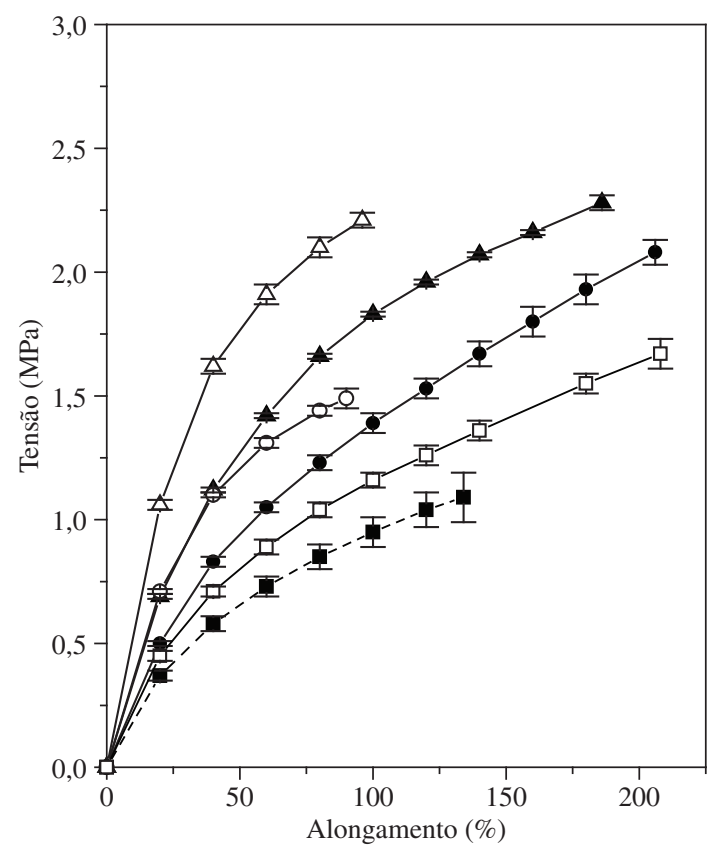

Figura 6. Curvas de tensão em função do alongamento obtidas para: (- $\mathbf{-}-)$ liner sem carga, (- $\square$-) liner/amianto, (- -) liner/SA 8, (- $\mathbf{-}$-) liner/SA 30, (-O-) liner/SA 8/GE 8, (- $\triangle$-) liner/SA 30/GE 8.

casos, apresentam-se entrelaçadas, o que torna sua dispersão menos eficiente, e gera pontos de fragilidade na matriz.

Quando se observa a curva de tensão do compósito contendo $8 \%$ de grafite expansível e $8 \%$ de silicato de alumínio nota-se que a os valores de tensão na ruptura e do alongamento são menores que os observados para o mesmo material na ausência das partículas de grafite, sendo que a tensão na ruptura diminui de 2,0 para 1,5 MPa, enquanto o alongamento é reduzido pela metade nos compósitos contendo o grafite expansível. A utilização de $8 \%$ de grafite expansível juntamente com $30 \%$ de silicato de alumínio causa a mesma redução nos valores de alongamento observados para o compósito contendo $8 \%$ de GE e SA, no entanto, os valores de tensão na ruptura são próximos, sendo 2,2 e 2,3 MPa para os compósitos com e sem grafite expansível, respectivamente. A redução nos valores de alongamento deve estar associada ao tamanho das partículas de GE ( 305 $\mu \mathrm{m})$, que sendo cerca de 27 vezes maiores que as partículas de silicato de alumínio, atuarão como pontos de fragilidade durante a solicitação do material. Já o fato do compósito contendo $8 \%$ de GE e $30 \%$ de SA mostrar valores de tensão na ruptura similares deve ser causado pelo maior reforço conferido à matriz pela utilização de $30 \%$ do silicato de alumínio, o que compensa a redução nos valores de tensão na ruptura causada pela introdução das partículas de grafite expansível.

\section{Conclusões}

Os resultados das análises termogravimétricas do poliuretano sem e com carga e os valores de energia de ativação para o início da decomposição, bem como os valores de coeficien- 
te de expansão térmica e de tensão e alongamento na ruptura indicam que o silicato de alumínio testado pode ser utilizado em substituição ao amianto com ganho nestas propriedades.

A utilização do grafite expansível causa um considerável aumento na energia de ativação para o início da decomposição térmica em amostras contendo $8 \%$ de SA, no entanto, observa-se que há uma perda significativa nos valores de alongamento na ruptura do material, sendo que para sua utilização estudos complementares devem ser realizados.

\section{Agradecimentos}

À Nacional de Grafite Ltda. e a Lamil Lage Minérios pelas doações das cargas. Aos Laboratórios PPM (AQI/IAE/ CTA) pelas análises mecânicas e AG (AQI/IAE/CTA) pelas análises granulométricas.

\section{Referências Bibliográficas}

1. Dreyfuss, P. "Polyurethane Precursor Resins" in: International Encyclopedia of Composites, vol. 4, p. 369, Lee, S. M. (ed.), VCH Publisher Inc., New York 1991.

2. Stover, E. R.; Juneau Jr., P. W. \& Brazel, J. P. - "Ablative Materials" in: Encyclopedia of Chemical Technology, vol. 1, p. 10, Mark, H. F.; Othmer, D. F.; Overberger, C. G.; Seaborg, G. T.; Grayson, M. \& Eckroth, D. (eds.), 3th. ed, John Wiley \& Sons, New York, 1978.

3. Gruntfest, I. J. - "Ablative Polymers", in: Encyclopedia of Polymer Science and Technology, vol. 1, p. 1, Mark, H.F., Gaylord, N.G., Bikales, N.M. (eds.), John Wiley \& Sons, New York, 1964.

4. Vaia, R. A.; Price, G.; Ruth, P. N.; Nguyen, H. T. \& Lichtenhan, J. - Appl. Clay Sci., 15, p. 67 (1999).

5. Tauzia, J-M. - "Thermal Insulations, Liners and Inhibitors" in: Solid Rocket Propulsion Technology, cap. 13, Davenas, A. (ed.), Pergamon Press Ltda., Grain Britain, 1993.

6. Chang, K. C.; Leung, C. C.; Tam, C. M.; Yu, W. C.; Hui, D. S. \& Lam, W. K. - Respiratory Medicine, 100, p.75 (2006).
7. Mendes, R., "Efeitos da Inalação de Fibras de Asbesto (Amianto) sobre a Saúde Humana: Estado Atual do Conhecimento e Fundamentação Científica para uma Política de Priorização da Defesa da Vida, da Saúde e do Meio-Ambiente". In: Instituto Brasileiro de Defesa ao Consumidor (IDEC). Brasília, 2000. Disponível em: http://www.idec.org.br/emacao.asp?id=767. Acesso em: 03 abril 2006.

8. Doðan, M. - Environ. Geol., 41, p. 571 (2002).

9. Albrecht, C.; Borm, P. J. A.; Unfried, K. - Mutation Research, 553, p.23 (2004).

10. Notícias do consumidor. In: Instituto Brasileiro de Defesa ao Consumidor (IDEC). 2005. Disponível em http:// www.idec.org.br/noticia.asp?id=4343. Acesso em 06 abril 2006.

11. Catálogo Lamil - Lages e Minérios - Linha Flexmil.

12. Streib, W. C., "Asbestos", in: Encyclopedia of Chemical Technology, vol. 3, p. 267, Mark, H. F.; Othmer, D. F.; Overberger, C. G.; Seaborg, G. T.; Grayson, M. \& Eckroth, D. (eds.), $3^{\text {th }}$. ed, John Wiley \& Sons, New York, 1978.

13. ASTM D 412 - 98a (reapproved 2002): Standard Test Methods for Vulcanized Rubber and Thermoplastic Elastomers Tension

14. Schartel, B., Braun, U., Schwarz, U., Reinemann, S. Polym., 44, p.6241 (2003).

15. Li, Z. \& Qu, B. - Polym. Degrad. and Stab., 81, p.401 (2003).

16. Xie, R. \& Qu, B. - Polym. Degrad. and Stab., 71, p. 375 (2001).

17. Osman, M. G.; Ploetze, M.; Suter, U. W. - J. Mater. Chem., 13, p.2359 (2003).

18. Torre, L.; Kenny, J. M. \& Maffezzoli, A. M. - J. Mater. Sci., 33, p. 3137 (1998).

19. ASTM E 1641-04: Standard Test Method for Decomposition Kinetics by Thermogravimetry.

Enviado: 16/02/06

Reenviado: 10/05/07

Aceito: 17/05/07 